\title{
No fruit fly an island?
}

Methods to study the behavior of Drosophila sp. in the context of a group may deepen our understanding of the neural mechanisms underlying social behavior.

\section{"No man is an island, entire of itself; every man is a piece of the continent, a part of the main."}

Almost half a century ago, Seymour Benzer settled on Drosophila as a genetically tractable and well-characterized organism in which to study animal behavior. The early efforts of Benzer and his team led to the isolation of mutants with disturbances in behaviors such as phototaxis, circadian rhythms and courtship, and the field of behavioral genetics was born.

The study of the genes and neural circuits that control the rich repertoire of behaviors in the fly continues today. However, the focus of these studies, by and large, is on the behavior of the individual fly (though often studied in populations for the sake of speed and statistics) or on pair-wise interactions in the study of aggression or of courtship.

In the case of Benzer's elegant countercurrent apparatus, for example, with which he isolated the first phototactic mutants, groups of flies are introduced into the apparatus, and the tendency of individual flies to move toward or away from a stimulus is scored. But the trajectories and detailed behaviors of individual flies in the context of the group cannot be monitored. In the paper first reporting the device, however, Benzer noted that flies are not entirely independent of other flies within a group (Benzer, S. Proc. Natl. Acad. Sci. USA 58, 1112-1119; 1967).

Although social interactions between fruit flies are probably not as extensive as those between eusocial insects such as ants and bees, interactions beyond fighting and mating are likely to be relevant. In the wild, fruit flies congregate on food sources and come into frequent contact both with members of their own and of other species. It is therefore quite likely that the brain of the fly evolved to interpret cues from its fellow flies, in addition to cues in its environment.

Indeed, some recent studies suggest that Drosophila behavior is influenced by the size and composition of the social group. The profile of sex pheromones on the cuticle of male flies, for instance, as well as the frequency of mating, are reported to be affected by genotypic composition of the social group, with males in heterogeneous groups mating more frequently (Krupp, J. et al. Curr. Biol. 18, 1373-1383; 2008).

The sleep patterns of flies are also influenced by social context. In a set of results that greatly tempt inappropriate anthropomorphizing, it has been reported that flies exposed to other flies sleep more (in the daytime) than those that are socially isolated; the strength of the effect depends upon the number of flies in the group and requires sensory input (Ganguly-Fitzgerald, I. et al. Science 313, 1775-1781; 2006). Several other behaviors-food searching, aggression, nocturnal locomotion and circadian rhythms - have also been reported to be modulated by exposure to other flies.

To learn how the Drosophila brain interprets social cues and uses them to modulate behavior, it would be of great value to observe the behavior of flies in groups and to do so quantitatively and in a manner amenable to statistical analysis. For a human observer, however, tracking the motion and actions of several flies over time, while keeping note of their individual identities, is, at best, a task of forbidding tediousness and arguably not possible in quantitative form at all. Two years ago, on this page, we highlighted the need for methodologies that allow rapid and high-throughput assessment of phenotypes in model organisms (Anonymous. Nat. Methods 4, 463; 2007); fruit fly social behavior is a particularly complex and challenging example of such phenotypes. Now, as reported by Michael Dickinson, Pietro Perona and colleagues in a paper published in this issue (p. 451) and discussed in a News and Views (p. 413), computer vision may be up to the job.

The automated system they present is able to track and behaviorally classify up to 50 unmarked flies interacting in an arena over several hours, and do so while keeping note of their individual identities with good accuracy. This should enable quantitative observations of fly behavior in the group context, and of how behavior is affected by interactions both within and between genotypes, and perhaps even between fly species. Coupled with the power of molecular genetic tools and the possibility to manipulate neuronal function in Drosophila, such observations could lead to a deeper understanding of the genes and neural circuits that control fly social behavior and potentially that of other insects as well.

Only time, and more experiments, will tell how rich the social repertoire of the fruit fly really is, and methods to carry out such experiments are therefore critical. Simple and genetically manipulable, yet complex enough to display interesting social behavior, the fly could be a route to a complete biological description of behavior. 